\title{
Inhomogeneous Magnetic Field Penetration in Superconducting Niobium Films
}

\author{
I.S. Abal'osheva ${ }^{a}$, A.V. Abal'oshev ${ }^{a}$, M.Z. CiePlak $^{a}$, L.Y. Zhu ${ }^{b}$ \\ AND C.-L. CHIEN ${ }^{b}$ \\ ${ }^{a}$ Instytut Fizyki PAN, al. Lotników 32/46, 02-668 Warszawa, Poland \\ ${ }^{b}$ The Johns Hopkins University, Baltimore, MD 21218, USA
}

\begin{abstract}
The peculiarities of magnetic field penetration in the form of fingering or dendritic instabilities are studied by magneto-optical technique in the niobium films of different thickness. It is observed that the reduction of $\mathrm{Nb}$ film thickness reduces the threshold field for instabilities, in agreement with the theoretical predictions. In addition, it is shown that the silver overlayer deposited on the top of $\mathrm{Nb}$ film leads also to the enhancement of instabilities, in marked contrast to the effect of gold or aluminum overlayers, which are known to suppress instabilities.
\end{abstract}

PACS numbers: 74.25.Ha, 74.25.Uv, 74.70.Ad

\section{Introduction}

The slow increase of the external magnetic field typically results in a gradual entry of the magnetic induction into type-II superconductor in the form of flux vortices. A critical state develops, characterized by a gradient of the vortex density which corresponds to the critical current density [1]. However, in thin superconducting films under certain conditions the critical state becomes metastable and the flux enters in form of flux avalanches which propagate much faster than the increase of the external magnetic field [2-4]. The origin of the avalanches is the local thermomagnetic instability. At low temperatures the vortices entering the sample are trapped by the pinning centers. With increasing external field the vortices trapped by the weakest pinning centers unpin, and their motion generates local heating in the area. This leads to the local decrease of the pinning force, and, in turn, to the unpinning of more vortices, producing the avalanche. In the presence of many similar pinning centers the precise location of the avalanche in the sample during subsequent magnetic field ramping is to a large extent random. It is important to mention that the flux exit during the lowering of the field may be strongly inhomogeneous as well, leading to "antiavalanche" behavior, when the flux remains trapped inside despite lowering of the external field, and then leaves suddenly from the large area. Such instabilities of magnetic flux can result in the noisy behavior of magnetization $[5,6]$ and they may lead to the suppression of the apparent critical current density $j_{\mathrm{c}}$ [7], affecting adversely the devices build on the base of the superconducting films. Therefore, the understanding of the avalanche phenomenon is very important for practical applications of films. In particular, it is important to establish how the avalanche propagation depends on sample thickness. Recently, a new theoretical description of the avalanche behavior has been proposed, and some comparisons of theory and experiments have been described $[8,9]$. However, the dependence of instabilities on the sample thickness has not been discussed yet. In the present work we study how the development of dendritic instabilities in thin niobium films is affected by the film thickness $t$, and by the deposition of silver overlayer on the top of the superconductor.

\section{Film preparation and measurement details}

Niobium films, of thickness between $100 \mathrm{~nm}$ and $1500 \mathrm{~nm}$, are grown by magnetron sputtering on glass substrates. The Ag layer on the top of Nb film, $150 \mathrm{~nm}$ thick, is deposited by thermal evaporation. A set of $\mathrm{Nb}$ films is shaped by photolithography into rectangles of dimensions $2 \times 2.5 \mathrm{~mm}, 5 \times 5 \mathrm{~mm}$ and $2 \times 6 \mathrm{~mm}$. The measurements are carried out in the magneto-optical setup consisting of the continuous-flow He cryostat, polarizing microscope, CCD camera and computer, in the temperature range between 4 and $8 \mathrm{~K}$ and in the magnetic field range between 0 and $500 \mathrm{Oe}$. As the magnetic field, perpendicular to the film plane, is slowly ramped from zero to $500 \mathrm{Oe}$, the flux penetration into the film is visualized. For visualization we utilize the Faraday effect in the Bi-doped ferrite garnet film placed directly on the top of the niobium film. In the Faraday effect the rotation of the plane of polarization of the light passing through the garnet sensor is proportional to the local magnetic field.

\section{Experimental results and discussion}

\subsection{Influence of the film thickness on the development of instability}

To quantify the effect of flux instabilities, we count the avalanches which are visible in the viewing area $5 \times 5 \mathrm{~mm}$ for any given magnetic induction $B$, sample temperature $T$, and sample thickness $t$. The results of the measurements are shown in Fig. 1. As the magnetic field 
is slowly increased, the first avalanches appear at some threshold value of the magnetic field $H_{\mathrm{th}}$. Further ramping of magnetic field results in the increase of the number of avalanches. Simultaneously, the uniform flux penetration is seen around the film edges. We observe that the increase of $t$ leads to the growth of the threshold magnetic field $H_{\mathrm{th}}$, and to the reduction of the number of avalanches which develop at any given $B$ and $T$.

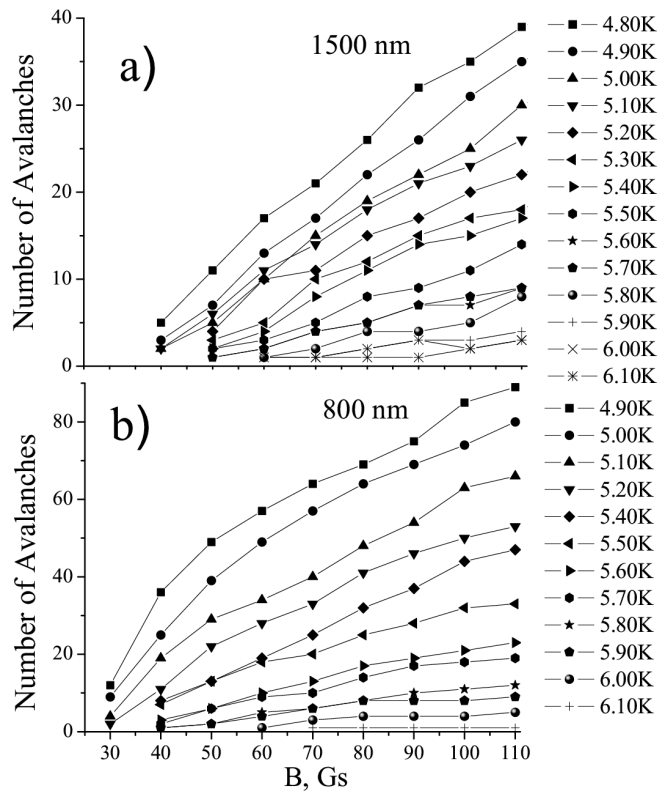

Fig. 1. Dependence of the number of avalanches on the magnetic field and temperature for Nb films with thicknesses $t=1500 \mathrm{~nm}(\mathrm{a})$ and $t=800 \mathrm{~nm}(\mathrm{~b})$

According to the theory [8], the dependence of the number of avalanches on $t$ is the result of competition of two mechanisms.

(i) The enhancement of instabilities with the reduction of $t$, due to nonlocality, which results in direct proportionality between $H_{\mathrm{th}}$ and $t$ (so that $H_{\mathrm{th}}$, as well as the critical electric field for development of avalanches, are both smaller in thin films than in bulk):

$$
H_{\mathrm{th}}=\left(\frac{j_{\mathrm{c}} t^{2}}{\pi w} \sqrt{\frac{k T^{*} j_{\mathrm{c}}}{E}}\right)^{1 / 2} .
$$

Here $w$ denotes in-plane dimension of the sample, and $T^{*}$ is the characteristic scale of the temperature dependence of $j_{\mathrm{c}}$.

(ii) The suppression of the instabilities with the reduction of $t$, due to thermal coupling between the film and the substrate.

In our films the mechanism (i) seems to prevail, which indicates that the thermal coupling of the $\mathrm{Nb}$ film to the glass substrate is most likely weak.

\subsection{Enhancement of instabilities by silver layer}

Figure 2 shows the result of covering of the niobium film with the thin Ag layer. We observe the enhancement of dendritic instabilities in the part of $\mathrm{Nb}$ film which is covered by Ag. The origin of this effect may be related to the increase of the surface pinning after Ag deposition. The increased surface pinning enhances the critical current density $j_{\mathrm{c}}$. This leads to the easier growth of the instabilities inside the sample, in accordance with the theoretical predictions [10]. This enhancement of instabilities remains in marked contrast to the suppression of instability observed for the superconducting films covered by the overlayers of $\mathrm{Au}$ [11] or $\mathrm{Al}$ [12]. In these cases the suppression of instability is explained by higher thermal conductivity of bilayer which improves the stability of the superconductor with respect to the thermal avalanches. Apparently, the surface pinning is affected differently depending on the type of covering layer. It seems that in the case of $\mathrm{Ag}$ layer on $\mathrm{Nb}$ the enhanced pinning dominates over the influence of metal layer on the thermal conductivity of the bilayer system.
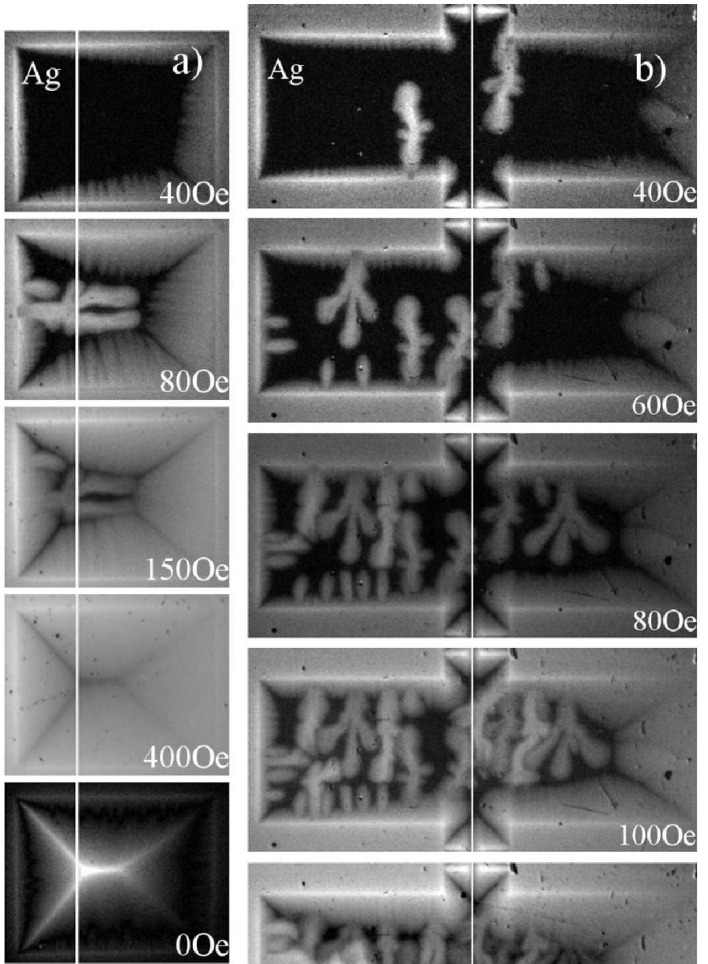

$\mathrm{Oe}$

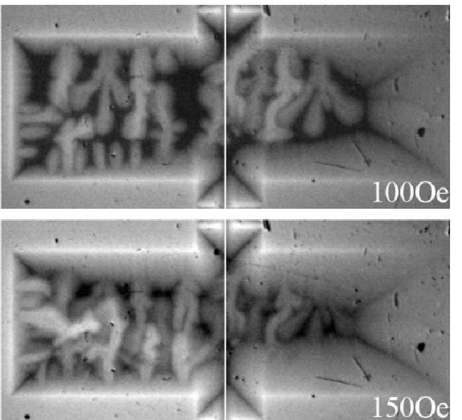

$\mathrm{T}=4,8 \mathrm{~K}$

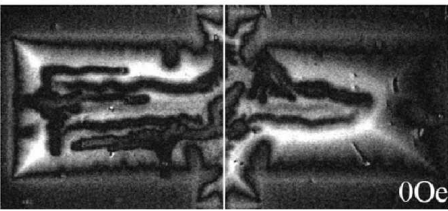

Fig. 2. The enhancement of instabilities by the silver overlayer in two $\mathrm{Nb}$ films. The white vertical line on the images denotes the boundary between covered and uncovered surface of $\mathrm{Nb}$ film. Ag layer is on the left side of the films, as denoted in the figure. Images taken at $H=0$ Oe show remanence state after the removal of the external magnetic field. 


\section{Conclusions}

The avalanche behavior in the superconducting $\mathrm{Nb}$ films deposited on the glass substrate has been studied for a series of films with different thicknesses, as well as for films covered with Ag overlayer. The thickness reduction leads to the enhancement of the instabilities, which indicates weak thermal coupling between $\mathrm{Nb}$ films and the glass substrate. Interestingly, the Ag overlayer also enhances instabilities. This suggests that the thermal coupling between $\mathrm{Nb}$ and the $\mathrm{Ag}$ is rather weak as well, so it cannot lead to the avalanche suppression, as observed, for example, for the $\mathrm{Au}$ or the $\mathrm{Al}$ overlayers on the superconducting films $[11,12]$. We explain this phenomenon by the Ag-induced increase of the surface pinning in $\mathrm{Nb}$ films, leading to the increase of the critical current density, and, as a consequence, to the easier development of avalanches.

\section{Acknowledgments}

This work was supported by Polish MNiSW grant N202 058 32/1202, and by NSF grants DMR05-20491 and DMR04-03849.

\section{References}

[1] C.P. Bean, Rev. Mod. Phys. 39, 41 (1968).

[2] E. Altshuler, T.H. Johansen, Rev. Mod. Phys. 76, 471 (2004).

[3] M. Baziljevich, C.M. Aegerter, R.J. Westerwaal, S. Enache, R.J. Wijngaarden, R. Griessen, Physica $C$ 406, 100 (2004).
[4] I.S. Aranson, A. Gurevich, V. Vinokur, Phys. Rev. Lett. 87, 067001 (2001).

[5] P. Espinazi, A. Setzer, D. Fuchs, Y. Kopelevich, E. Zeldov, C. Assmann, Phys. Rev. B 60, 12454 (1999).

[6] Z.W. Zhao, S.L. Li, Y.M. Ni, H.P. Yang, Z.Y. Liu, H.H. Wen, W.N. Kang, H.J. Kim, E.M. Choi, S.I. Lee, Phys. Rev. B 65, 064512 (2002).

[7] A.V. Bobyl, D.V. Shantsev, T.H. Johansen, W.N. Kang, H.J. Kim, E.M. Choi, S.-I. Lee, Appl. Phys. Lett. 80, 4588 (2002).

[8] D.V. Denisov, A.L. Rakhmanov, D.V. Shantsev, Y.M. Galperin, T.H. Johansen, Phys. Rev. B 73, 014512 (2006).

[9] D.V. Denisov, D.V. Shantsev, Y.M. Galperin, EunMi Choi, Hyun-Sook Lee, Sung-Ik Lee, A.V. Bobyl, P.E. Goa, A.A.F. Olsen, T.H. Johansen, Phys. Rev. Lett. 97, 077002 (2006).

[10] I.S. Aranson, A. Gurevich, M.S. Welling, R.J. Wijngaarden, V.K. Vlasko-Vlasov, V.M. Vinokur, U. Welp, Phys. Rev. Lett. 94, 037002 (2005).

[11] E.-M. Choi, Hyun-Sook Lee, Hyun Jung Kim, Byeongwon Kang, Sung-Ik Lee, A.A.F. Olsen, D.V. Shantsev, T.H. Johansen, Appl. Phys. Lett. 87, 152501 (2005).

[12] M. Baziljevich, A.V. Bobyl, D.V. Shantsev, E. Altshuler, T.H. Johansen, S.I. Lee, Physica $C$ 369, 93 (2002). 